\title{
Medienbildung in der frühen Kindheit
}

\author{
* Zurück zur Übersicht über den \\ Themenschwerpunkt
}

Die Landesgruppe Baden-Württemberg der GMK (Gesellschaft für Medienpädagogik und Kommunikationskultur e.V.) veröffentlichte im April 2008 eine Stellungnahme „Zur Situation und Perspektiven der Medienbildung in Baden-Württemberg". Darin wird in einem Teil 2, der federführend von Dr. Ulrike Bischof (Stuttgart) und Anne Radlinger (Ulm) formuliert wurde, auf die Situation der Medienbildung im Kindergarten eingegangen, ergänzt um konkrete Forderungen zur Verbesserung der Situation. Leider hat sich seit April 2008 nichts verändert - die Forderungen sind nach wie vor aktuell. Wir veröffentlichen im Folgenden den entsprechenden Teil aus der GMK-Stellungnahme.

Die Redaktion

\section{Stellungnahme der GMK- Landesgruppe Baden- Württemberg zur Medienbil- dung im Kindergarten \\ Medienbildung im Kindergarten}

Wir begrüßen, dass die Landesregierung Baden-Württembergs verstärkt in der frühen Bildung ansetzen und die Basiskompetenzen von Vorschulkindern fördern will.

Wissenschaft und Gesellschaft sind einig, dass eine gedeihliche Entwicklung von Kindern frühe Bildung und Förderung voraussetzt und in den ersten Lebensjahren dafür und ebenso für die Sprachentwicklung Grundlagen geschaffen werden müssen. Im Orientierungsplan Bildung und Erziehung für die baden-württembergischen Kindergärten widerspiegeln sich diese Erkenntnisse, weniger jedoch die Lebenswelt und die unterschiedlichen sozialen und kulturellen Bedingungen, unter denen Kinder in die Gesellschaft hineinwachsen. Auf diese Lebenswelt wird lediglich kurz Bezug genommen, wenn es heißt: „Eine ästhetischkünstlerische Bildung setzt bei der Lebenswirklichkeit an, die Kindern bedeutsam erscheint" oder geschrieben wird: „Kinder nehmen Bilder aus Alltag, Kunst und Medien bewusst wahr und setzen sich damit auseinander." Damit wird weder die Medienrealität noch die Diskrepanz zwischen ihr und dem Wissen von Erzieherinnen und Eltern angemessen berücksichtigt.

Wenn in einem „Kinderland“ BadenWürttemberg an der Lebenswirklichkeit der
Kinder angesetzt werden soll, dann gehört dazu, dass die von ihnen genutzten Medien mit ihren Chancen und Gefahren schon im Kindergarten thematisiert werden. Rolle und Verantwortung der Erzieherinnen in den Kindergärten und -tagesstätten sind dabei hoch, denn in vielen Elternhäusern findet Medienerziehung aus Unkenntnis oder Verunsicherung kaum statt. Folglich muss auch außerhalb der Familie gesorgt werden, dass Kinder aller Schichten und Milieus die Vielfalt des medialen Angebots kennen- und kreativ anwenden lernen. Damit das geschehen kann, ist ein geschultes Personal notwendig, das dafür eine solide Grundlagenausbildung erhalten hat und dem Gelegenheit zur Fort- und Weiterbildung in einer Weise geboten wird, die sie auch befähigt, ihr Wissen an Eltern weiterzugeben. Dies wiederum setzt eine angemessene Personal- und Materialausstattung der Einrichtungen voraus.

Indessen sind Entwicklungen feststellbar, die teils gegenüber dem Erreichten einen Rückschritt darstellen, wovon insbesondere die Streichung des Faches Kinder- und Jugendliteratur/Medienpädagogik in den Lehrplänen der Fachschulen bzw. in der Ausbildung von Erzieherinnen zeugt und ebenso die nur punktuelle, projektbezogene Förderung von erfolgreichen, preisgekrönten Weiterbildungsmaßnahmen (z.B. die zertifizierte Qualifizierung zur Fachkraft für Medienpädagogik im Kindergarten der Familien-Bildungsstätte Ulm e.V.) u.a. sporadische, projektbezogene Angebote.

Die Landesgruppe der GMK BadenWürttemberg fordert daher:

1. Kindergärten, Horte und Vorschuleinrichtungen sollten sich verstärkt für medienpädagogische Projekte öffnen, die Medienkompetenz früh fördern, handlungsorientiert sind und auch dadurch zur kulturellen Bildung der Kinder beitragen.

2. Wissen über Medien ist ein eigenständiger Bildungsbereich. Die Förderung der Medienkompetenz ist in den Bildungsplan für die Kindergärten angemessen $\mathrm{zu}$ integrieren und wie die anderen Bildungsbereiche mit Umsetzungsvorschlägen und Zielvorstellungen zu versehen. 
3. Medienpädagogik muss in den Lehrplänen der Fachschulen als selbständiges und obligatorisches Fach erhalten beziehungsweise in die Curricula der Hochschulen integriert werden und es müssen verpflichtende Fortbildungsangebote gewährleistet werden. 